\title{
Electron density fluctuations accelerate the branching of positive streamer discharges in air
}

\author{
A. Luque ${ }^{1}$ and $U$. Ebert ${ }^{2,3}$ \\ ${ }^{1}$ Instituto de Astrofísica de Andalucía (IAA), CSIC, P.O. Box 3004, 18080 Granada, Spain \\ ${ }^{2}$ Centrum Wiskunde \& Informatica (CWI), P.O. Box 94079, 1090 GB Amsterdam, The Netherlands \\ ${ }^{3}$ Department of Physics, Eindhoven University of Technology, P.O. Box 513, 5600 MB Eindhoven, The Netherlands
}

(Received 8 February 2011; revised manuscript received 26 September 2011; published 25 October 2011)

\begin{abstract}
Branching is an essential element of streamer discharge dynamics. We review the current state of theoretical understanding and recall that branching requires a finite perturbation. We argue that, in current laboratory experiments in ambient or artificial air, these perturbations can only be inherited from the initial state, or they can be due to intrinsic electron-density fluctuations owing to the discreteness of electrons. We incorporate these electron-density fluctuations into fully three-dimensional simulations of a positive streamer in air at standard temperature and pressure. We derive a quantitative estimate for the ratio of branching length to streamer diameter that agrees within a factor of 2 with experimental measurements. As branching without this noise would occur considerably later, if at all, we conclude that the intrinsic stochastic particle noise triggers branching of positive streamers in air at atmospheric pressure.
\end{abstract}

DOI: 10.1103/PhysRevE.84.046411

PACS number(s): 52.80.Mg, 82.20.Wt

\section{INTRODUCTION}

\section{A. Observations of branching streamers}

Streamers are filamentary electrical discharges that propagate through a nonconducting medium when it is suddenly exposed to a high electric field [1]. As first stages of electrical breakdown [2], they are found in nature preceding a lightning stroke and as the building blocks of upper-atmospheric discharges above thunderstorms [3-6]. Due to their efficient production of chemical radicals [7], streamers are used in industry for gas cleaning and sterilization.

An essential element of the electric breakdown in large volumes is that streamers appear not alone but in so-called coronas, e.g., in the streamer corona of lightning leaders or in corona reactors. Frequently, they form irregular trees with many branches. Branching is an essential element in the formation of the discharge trees, and these branching events have now been characterized in detail in the upper-atmospheric discharges called sprites [8] as well as in laboratory experiments on positive streamers emitted from needle electrodes. In the laboratory, branch lengths and branching angles were measured in air at varying densities $[9,10]$, the large influence of the nitrogen-oxygen ratio on the branching and avalanche structure was investigated in detail [11], and semiquantitative explanations of streamer diameters, velocities, and avalanche structures in these experiments were provided [12,13]. However, a quantitative understanding of the observed branching structures is not available yet. First, quantitative theoretical results will be presented in the present paper.

\section{B. Present understanding of streamer branching}

\section{Relation between ionization avalanches and streamer branches}

The old concept of streamer branching due to avalanches [14-16] can be found in many textbooks and is in continued use in the current literature [17]; it goes back to Raether [1] (where in most cases the English translation of Raether's ideas by Loeb and Meek [18] is quoted): photoionization supplies single electron-ion pairs ahead of the head of a positive streamer, these electrons create ionization avalanches that run backward toward the streamer, and those avalanches then develop into new streamer branches. Wormeester et al. [13] provide estimates for the density of these avalanches in gases with different nitrogen-oxygen ratios. These estimates show that avalanches created by single electrons can be visible in recent experiments in nitrogen with 1 part per million (ppm) oxygen [11] but that the number of avalanches in air is so large that they cannot be distinguished visually. Therefore there is certainly no direct relation between ionization avalanches and streamer branches. This is also physically plausible: an avalanche can only develop into a streamer branch if an ionization and space-charge wave runs forward into the avalanche trail and transforms the backward propagating avalanche into a forward running streamer.

\section{Metastability of streamers}

Simulations [19-21] combined with the analysis of a reduced moving-boundary streamer model [22-26] have shown that streamers can branch even in fully deterministic density models through a Laplacian instability; this mechanism is similar to the one underlying the branching of viscous fingers in two-fluid Hele-Shaw flow [27,28]. This instability can develop when the space-charge layer around the streamer head is much thinner than the streamer radius [26]. In the extreme case of a planar streamer ionization front, an infinitesimally small perturbation is sufficient to trigger a branching instability $[29,30]$, but for an ionization front curved around a propagating streamer head a finite perturbation is necessary [24,25], even if the stabilizing effects of electron diffusion and photoionization are neglected. This is because small perturbations of curved propagating fronts are convected to the side of the structure while they are growing; therefore only perturbations of finite size have a chance to grow out into a self-sustaining nonlinear branch structure at the streamer head. In this way, streamer fingers are similar to laminar pipe flow: they are never completely unstable. Even if they are in a metastable state that is susceptible to branching or to the transition to turbulence, they need a finite perturbation to transit to the new dynamic state. 


\section{Possible triggers of the branching instability}

(a) Initial conditions. The finite perturbation that triggers branching can be inherited from some earlier stage of evolution or, in particular, from the initial condition of a simulation. From this initial state the streamer then develops in a completely deterministic manner into a branching state. This behavior was seen in the simulations of a density model for negative streamers in pure nitrogen by Montijn et al. [20] where the branching time converged when simulations were performed on an increasingly fine numerical grid. How branching is triggered by initial conditions was recently studied in greater detail in the framework of a moving boundary approximation for streamer fronts by Kao et al. [25]. Though authors earlier expressed doubts on this mechanism [31,32], the mathematical correspondence between streamer branching and well-investigated moving boundary problems with Laplacian instabilities leaves no doubt that this branching mechanism works.

(b) Numerical perturbations. In other simulations of the fully deterministic streamer density model [19,21], a coarser numerical grid can have created additional perturbations that probably have accelerated the branching instability. It should be noted though that the overall structure of the developing branching instability is essentially the same.

(c) Macroscopic external perturbations. Macroscopic external perturbations of the medium through which a streamer propagates can change its path and lead to branching. Many external perturbations are possible; one can think of dust particles in air or of air bubbles in water or of other macroscopic objects, of a variation of air densities (e.g., due to locally increased temperature or due to gravity waves and turbulence in high atmospheric layers), of a variation of gas compositions, or of patches of higher ionization density [33] (due to external radiation sources or due to previous discharges). An amusing experimental result showing the effect of the traces of a previous discharge on the next one can be found in Ref. [34]. A related effect can be seen in Ref. [35], where two neighboring streamers approach each other when the attraction of the jointly created high photoionization density between them overcomes their electrostatic repulsion. The simulations of Papageorgiou et al. [36] show a similar deviation of the streamer path, here due to a variation of the medium density. Babaeva and Kushner found already earlier that a medium density variation can also make a streamer branch $[37,38]$. In their case, the streamer is attracted to several air bubbles in water (that are modeled as regions of lower medium density) that have a similar diameter as the streamer itself. As the reduced electric field $E / N$ is higher in a lower medium density $N$, streamers preferably run through the bubbles and branch in order to pass through several ones. A common denominator of these observations is that the external perturbations to which the streamer is attracted are at least as large as the streamer diameter.

\section{Irrelevance of macroscopic perturbations for streamer branching in ambient air}

Macroscopic perturbations are certainly not the cause of streamer branching in the experiments described in Refs. [11,39]. These experiments are performed in a steel vacuum vessel without plastic parts that was baked out to remove all traces of water and other volatile components, and it was pumped down several times to remove any traces of other gases; the streamers then grow through continuously refreshed nitrogen or artificial air (which is an oxygen:nitrogen mixture in the ratio of 20:80) with impurity rates well below $1 \mathrm{ppm}$. We refer, in particular, to those experiments in Refs. [11,39] where voltage pulses were applied only every 10 or $100 \mathrm{~s}$. In this case, the results agree with those in a completely fresh gas fill, and all remaining ionization and space-charge effects of previous discharges disappear.

But the structure of branching streamers in ambient air in Refs. [10,40,41] can hardly be distinguished from that in artificial air. This means that macroscopic perturbations can also be excluded as a trigger of streamer branching in the experiments in ambient air presented in Refs. [10,40,41].

Therefore streamer branching instabilities in ambient room air can only be triggered by initial conditions or by intrinsic noise. The irregularity of the branching structures hints at some stochastic mechanism.

\section{Intrinsic fluctuations, avalanches, and their modeling}

Electrons are not a continuous density, but quantized. Ions and neutral molecules are discrete particles as well. In regions where their number densities are high, a density model can be a valid and numerically efficient approximation. In the streamer interior, the densities of charged particles are sufficiently high to justify a density approximation, and we recall that the density of neutral molecules is even several orders of magnitude larger. But the density approximation for streamer discharges breaks down, in particular, ahead of the ionized channel, if electron number densities are too low or if electric fields are too high. For a general discussion of these features, including electron runaway from negative streamers, we refer to Ref. [42] and references therein.

(a) Monte Carlo and hybrid models. The distribution of positions and energies of electrons is naturally included in Monte Carlo models [42-47] that follow the single electron motion. Those models are microscopically very accurate at the cost of high computational demands. Even state-of-the art spatially hybrid codes $[42,46,48]$ are still limited to quite short streamers, and simulations of branching streamers with such codes are not available yet.

(b) Other models with fluctuations. Arrayás et al. [49] and Pancheshnyi suggested other models that include electrondensity fluctuations in some manner. Arrayás et al. [49] inserted electrons at random ahead of a planar streamer ionization front. As discussed correctly in their Sec. 7 (but not in the abstract, introduction, or conclusion), these electrons grow out into new avalanches as the electric field ahead of a planar front has to stay above the breakdown value. The addition of any electrons ahead of such a front, whether in a deterministic or in a stochastic manner, will make a planar front move faster, as already discussed in Refs. [50,51]. Pancheshnyi simulated positive streamers where photoionization created electrons at random positions ahead of the streamer front while the created ionization avalanches were treated with a density model. However, these results are not published, and to the best of our knowledge they are also not available in conference proceedings. For published results on ionization 
avalanches in overvolted gaps (i.e., where the electric field is above the breakdown value), we refer to Ref. [47] and to the present paper.

\section{Content of the paper}

Here we introduce a new computational model that at all times takes the randomness of the positions of the discrete electrons into account while assuming that the local-field approximation for the electron energies is sufficient; therefore we do not account for the full electron energy distribution, with possible runaways, but we accurately model the intrinsic electron-density fluctuations. We present its quantitative predictions for positive streamers in air at standard temperature and pressure.

The model is a spatially extended stochastic model [52]. Since particles are not individually tracked, the required memory and computations are roughly independent of the number of active particles, in contrast to Monte Carlo methods. We study the evolution toward branching with realistic density fluctuations in full three dimensions. We find that the instability develops faster than in a fully deterministic density model. Through extrapolation of the numerical results, we obtain branching ratios that are consistent with the experiments in Refs. [9,10]. This suggests that the branching of positive streamers in air at atmospheric pressure is triggered by the intrinsic electron-density fluctuations.

\section{MODEL}

\section{A. Density model}

The most relevant microscopic processes in streamers are two-body reactions between free electrons and neutral gas molecules. Therefore most quantities of a streamer discharge scale with the neutral gas density in a definite manner called Townsend scaling [6]. Following Ref. [53] we define a typical length for streamers in air $l_{0} \approx 2.3 \mu \mathrm{m} \cdot\left(N_{0} / N\right)$, a typical electric field $E_{0} \approx 2 \cdot 10^{5} \mathrm{~V} / \mathrm{cm} \cdot\left(N_{0} / N\right)$, a typical time $t_{0}=3 \cdot 10^{-12} \mathrm{~s} \cdot\left(N_{0} / N\right)$, and a typical density of charge carriers $n_{0} \equiv \epsilon_{0} E_{0} / \mathrm{e} l_{0} \approx 4.7 \cdot 10^{14} \mathrm{~cm}^{-3} \cdot\left(N_{0} / N\right)^{2}$, where e is the elementary charge, $N$ is the molecule number density of air, and $N_{0}$ is the number density at standard temperature and pressure, used as an arbitrary reference. We refer to Ref. [6] for a physical interpretation of these quantities and scaling laws.

Using these typical magnitudes one can build a dimensionless streamer model. We consider here a model for air $[12,53]$ that includes photoionization [54]. We are interested in the dynamics of the streamer head, where impact ionization strongly dominates over electron removal by attachment and the latter can be safely neglected. The governing equations in dimensionless form are therefore

$$
\begin{aligned}
\partial_{t} \sigma & =D \nabla^{2} \sigma+\nabla \cdot(\sigma \mathbf{E})+S^{(\mathrm{ph})}+S^{(\text {impact })}, \\
\partial_{t} \rho & =S^{(\mathrm{ph})}+S^{(\mathrm{impact})}, \\
\nabla^{2} \phi & =\sigma-\rho, \quad \mathbf{E}=-\nabla \phi .
\end{aligned}
$$

Here $\sigma$ and $\rho$ are the (dimensionless) electron and ion densities; $S^{(\mathrm{ph})}$ and $S^{\text {(impact) }}$ are, respectively, the photoionization and impact ionization sources of electron-ion pairs; $\phi$ is the electrostatic potential; $\mathbf{E}$ the electric field; and $D$ is a diffusion coefficient, taken as $D=0.1$.

There are several corrections to Townsend scaling [6]. One arises from collisional quenching of photoionization [21], here included in $S^{(\mathrm{ph})}$, which contains an explicit dependence on the air density. A second correction, on which we focus here, arises from the finite number of particles and the stochastic nature of microscopic processes; this cannot be expressed in Eqs. (1)-(3), because they only contain macroscopic quantities. Rather we will take a discretization of Eqs. (1)-(3) and convert it into a spatially extended stochastic model [52].

\section{B. Modeling the intrinsic density fluctuations}

Since the importance of stochastic noise depends on the particle number density, our first step is to derive, from the magnitudes described above, a dimensionless parameter for the typical number of charge carriers contained in a typical volume. We define $g \equiv n_{0} l_{0}^{3}=E_{0} \varepsilon_{0} l_{0}^{2} /$ e. This is the number of elementary charges that has to sit in each area $l_{0}^{2}$ of an infinite charged plane to create a jump $E_{0}$ in the electric field. In air $g \approx 5700 \cdot\left(N_{0} / N\right)$; at an altitude of $70 \mathrm{~km}$, typical for sprites, $g \approx 10^{8}$.

The relative amplitude of the statistical fluctuations of a number $g$ of particles is $g^{-1 / 2}$. The limit of negligible fluctuations is $g^{-1 / 2} \ll 1$. In air at atmospheric pressure, $g^{-1 / 2} \approx 0.01$ and stochastic noise is a relatively small correction on the fluid description. However, as we will see, due to the strongly nonlinear nature of streamer discharges, such small fluctuations can be amplified by strong electric fields and alter significantly the propagation of a streamer. For sprites, $g^{-1 / 2}$ is much smaller, about $10^{-4}$.

Now let us take a spatial discretization of Eqs. (1)-(3): the simulation domain (see Fig. 1) is divided into cells $C_{i}$, each with a dimensionless volume $v_{i}=V_{i} / l_{0}^{3}$ ( $V_{i}$ is the dimensional volume). If we are given the dimensionless densities in each cell, $\sigma_{i}$ and $\rho_{i}$, we know, from our discretization, how to calculate the left-hand sides of Eqs. (1)-(3). In particular, we can calculate the source term $S_{i}=S_{i}^{(\mathrm{ph})}+S_{i}^{\text {(impact) }}$ and the flux from cell $C_{i}$ to each neighboring cell $C_{j}$, which we denote $F_{i j}$.

But instead of densities one has a discrete number of electrons and ions in each cell, $N_{i}^{(e)}$ and $N_{i}^{(i)}$; the dimensionless
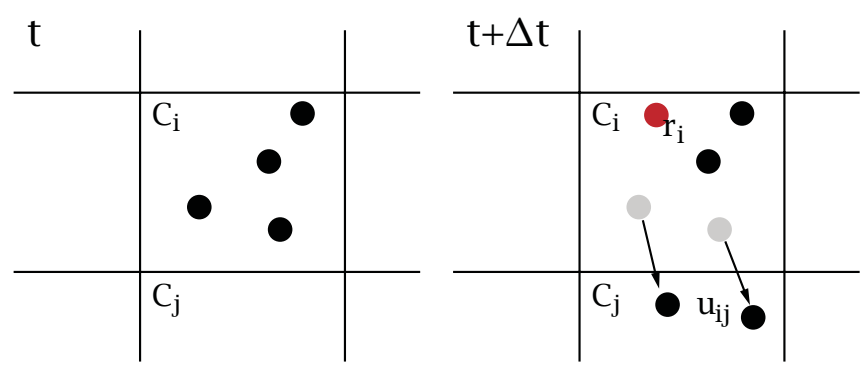

FIG. 1. (Color online) Scheme of an elementary time step in the lattice model. Each electron is represented here by a dot; note, however, that we do not keep track of every individual particle, but only of the number of particles in each cell. During the time step from $t$ to $t+\Delta t$ we move $u_{i j}$ electrons from cell $C_{i}$ to $C_{j}$. Meanwhile, $r_{i}$ electrons arise in $C_{i}$ from impact ionization and photoionization. 
densities are therefore

$$
\begin{aligned}
\sigma_{i} & =N_{i}^{(e)} / n_{0} V_{i}=N_{i}^{(e)} / g v_{i}, \\
\rho_{i} & =N_{i}^{(i)} / g v_{i} .
\end{aligned}
$$

We may now use these to calculate the source terms and the fluxes. The problem is now how to update the number of particles as time evolves. To simplify the description, let us first describe a time stepping that will tend to a forward Euler discretization with time step $\Delta t$.

We look first at the source terms. Let $r_{i}$ be the number of electron-ions pairs created in $C_{i}$ during a time $\Delta t$. This quantity follows a Poisson distribution with average $\lambda_{i}=$ $g v_{i} S_{i} \Delta t$. So in the simulation we draw for each $i$ a sample from the Poisson probability distribution:

$$
p\left(r_{i}\right)=\lambda_{i}^{r_{i}} e^{-\lambda_{i}} / r_{i} !
$$

The transport terms are slightly more complicated because the number of electrons must be conserved. We interpret the $g v_{i} F_{i j} \Delta t$ as the average number of electrons that flows from $C_{i}$ to $C_{j}$ during $\Delta t$ (note that $F_{i j} \neq F_{j i}$ ). Thus the probability for an electron in $C_{i}$ at $t$ to end in $C_{j}$ at $t+\Delta t$ is $p_{i j}=g v_{i} F_{i j} \Delta t / N_{i}^{(e)}$ for $i \neq j$. But since the electron must end somewhere, ${ }^{1}$

$$
p_{i i}=1-\sum_{j \neq i} p_{i j} .
$$

We can use these $p_{i j}$ to obtain the number of electrons moving from $C_{i}$ to $C_{j}$, that we denote $u_{i j}$. The probability distribution for the electrons exiting $C_{i}$ is the multinomial distribution with $\sum_{j} u_{i j}=N_{i}^{(e)}$.

Now we have all the ingredients to update the particle numbers as

$$
N_{i}^{(e)}(t+\Delta t)=N_{i}^{(e)}(t)+r_{i}+\sum_{j}\left(u_{j i}-u_{i j}\right) .
$$

As mentioned above, this scheme tends to an explicit Euler time discretization of the fluid equations as $g^{-1 / 2} \rightarrow 0$. But we can also design a two-step time updating that tends to secondorder Runge-Kutta [55] if we (a) use the particle numbers at $t$ to calculate $S_{i}(t)$ and $F_{i j}(t)$, (b) perform a half-step using $\Delta t / 2$ to update the particle numbers, (c) use the new particle numbers to obtain $S_{i}(t+\Delta t / 2)$ and $F_{i j}(t+\Delta t / 2)$, and (d) define $S_{i}^{\prime}(t)=\left[S_{i}(t)+S_{i}(t+\Delta t)\right] / 2$ and $F_{i j}^{\prime}(t)=\left[F_{i j}(t)+\right.$ $\left.F_{i j}(t+\Delta t)\right] / 2$ and use them to perform a step $\Delta t$. Note that in principle step (b) could also implement a standard continuum step, since nothing forces us to preserve an integer number of particles in that intermediate step; we opted however to use the same stochastic step in both stages of the algorithm.

We have not yet mentioned the spatial discretization to calculate $F_{i j}$. The reason is that the scheme is flexible on that. We used here the scheme described in Ref. [55]. This is a flux-limited, nonlinear discretization scheme and it poses an additional difficulty: it sometimes leads to negative $F_{i j}$ which cannot be interpreted as a probability. In that case, we

\footnotetext{
${ }^{1}$ Note that nothing assures us that $p_{i i}>0$, although it would be if $\Delta t \rightarrow 0$. The solution that we implemented in that case is to set $p_{i i}=0$ and renormalize the rest of the $p_{i j}$ such that $\Sigma_{j} p_{i j}=1$.
}

rearrange the fluxes by letting $F_{i j} \rightarrow F_{i j}^{\prime}$, with $F_{i j}^{\prime}=0$ and $F_{j i}^{\prime}=F_{j i}-F_{i j}$, until no negative fluxes remain.

\section{RESULTS}

\section{A. Simulating avalanches and streamers in an overvolted gap}

We now use the adaptive grid refinement and the fluxes and reaction terms from Ref. [55] and the three-dimensional cylindrical mesh of Ref. [35]. As a first application, let us analyze the initiation of breakdown in a small plane-to-plane geometry with a potential difference of $16 \mathrm{kV}$ between two electrodes separated by $2 \mathrm{~mm}$ of air at standard temperature and pressure. This means that the discharge develops in a background electric field of $80 \mathrm{kV} / \mathrm{cm}$, which is well above the breakdown value. The simulated volume is discretized into cells $\Delta r=\Delta z=8 \mu \mathrm{m}$ and $\Delta \theta=2 \pi / 64$. As an initial condition, we set a neutral hemispherical Gaussian seed at $z=r=0$ (positive electrode) containing $\sim 6 \cdot 10^{5}$ electrons.

Figure 2 shows the evolution of a cross-section of the electron densities up to $1.35 \mathrm{~ns}$. In that short time span, photoionization has seeded a multitude of avalanches all across the simulation volume. A very similar evolution is observed in the Monte Carlo simulations of Refs. [42,47], where the

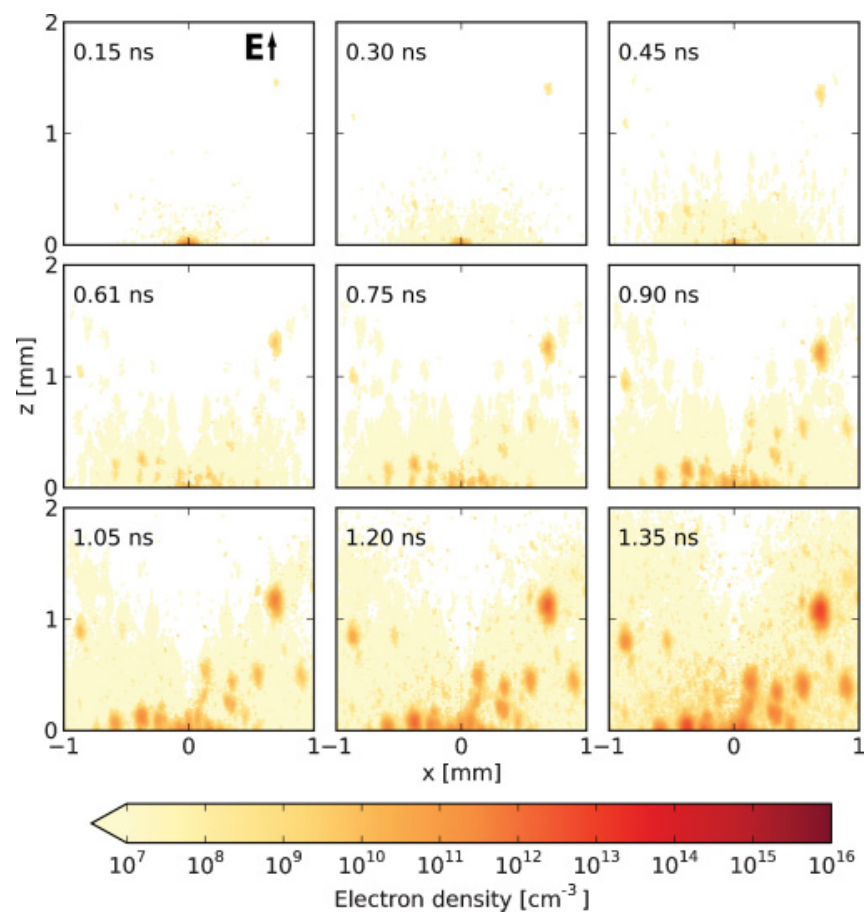

FIG. 2. (Color online) Snapshots of the cross section of the electron density in the $y=0$ plane in a 2-mm gap between planar electrodes with a potential difference of $16 \mathrm{kV}$; i.e., the background field of $80 \mathrm{kV} / \mathrm{cm}$ is well above the breakdown value. We plot the densities of the simulation cells centered at $y=0$ with coordinates $x, z$ : the volume of those cells is proportional to their separation from the axis, and therefore in this cross section the relative fluctuations are larger close to the $x=0$ line. Photoionization creates multiple electron-ion pairs throughout the volume, and the electrons start avalanches. Therefore the electric breakdown extends over the complete volume and no actual streamer is initiated. A movie of this simulation is available in the supplementary material [56]. 
discharge evolution in ambient air in a gap of $1.17 \mathrm{~mm}$ in a background field of $100 \mathrm{kV} / \mathrm{cm}$ is followed for $0.7 \mathrm{~ns}$; the initial condition consisted of 500 electrons and ions close to the cathode. In both cases, the length over which photoionization can create new electron-ion pairs is comparable to the size of the simulated system. Therefore there is a strong competition between uniform breakdown and spatially structured streamer formation. A similar evolution probably takes place in the overvolted region around a strongly curved needle or wire electrode, if the voltage is applied sufficiently rapidly. Although numerical simulations sometimes use overvolted gaps to investigate the propagation of positive streamers [57,58], we have shown here that fluctuations in the production of photoelectrons must be included in more realistic simulations.

We remark that the similarity of the evolution in Li's Monte Carlo model [42,47] and in the present density-fluctuation model show that the physics of electron-density fluctuations was correctly imbedded in both of these models.

\section{B. Simulating streamers in an undervolted gap}

A homogeneous breakdown cannot occur when the background field is below the breakdown value. In this case, electric breakdown can only occur through the field enhancement at the tip of a streamer or of a pointed electrode.

We therefore now run a simulation where a needle is inserted as a protrusion into one of the planar electrodes; the numerical implementation is discussed in Ref. [58]. The needle carries a positive voltage of $10.5 \mathrm{kV}$, it is $2 \mathrm{~mm}$ long, and its tip is separated from the plate by $7.2 \mathrm{~mm}$; therefore the average background field between the planar electrodes is $11.4 \mathrm{kV} / \mathrm{cm}$, well below the breakdown value while breakdown is possible close to the needle tip. As an initial condition we set a semispherical neutral Gaussian ionization seed at the needle tip with a radius of $73.6 \mu \mathrm{m}$ and a peak ionization density of $4.7 \cdot 10^{18} \mathrm{~cm}^{-3}$. We used an adaptive refinement strategy [55] with coarsest grid $\Delta z=\Delta r=40 \mu \mathrm{m}$ and finest grid $\Delta z=$ $\Delta r=2.5 \mu \mathrm{m} .^{2}$ Since full three-dimensional simulations are too demanding, we chose to run the simulation with cylindrical symmetry and $g^{-1 / 2}=0$ up to $t=13.5 \mathrm{~ns}$. Then we remove the constraint of cylindrical symmetry and introduce stochastic noise at the level expected at atmospheric pressure $(g=5700)$. The continuous density at each cell is then interpreted as an average and discrete numbers of particles are obtained by drawing random samples from a Poisson distribution with this average.

To represent the evolution of this three-dimensional simulation while it deviates only slightly from cylindrical symmetry, let us consider the average of some quantity around the azimuthal angle

$$
\langle u\rangle_{\theta}=\frac{1}{2 \pi} \int_{0}^{2 \pi} u(r, z, \theta) d \theta .
$$

\footnotetext{
${ }^{2}$ In the adaptive refinement algorithm one has to interpolate densities from a coarser to a finer grid. We adapted this to our discrete algorithm by interpreting densities as probabilities and again sampling from a multinomial distribution. Thus, all cells contain a discrete number of particles and this number is preserved across nested grids.
}
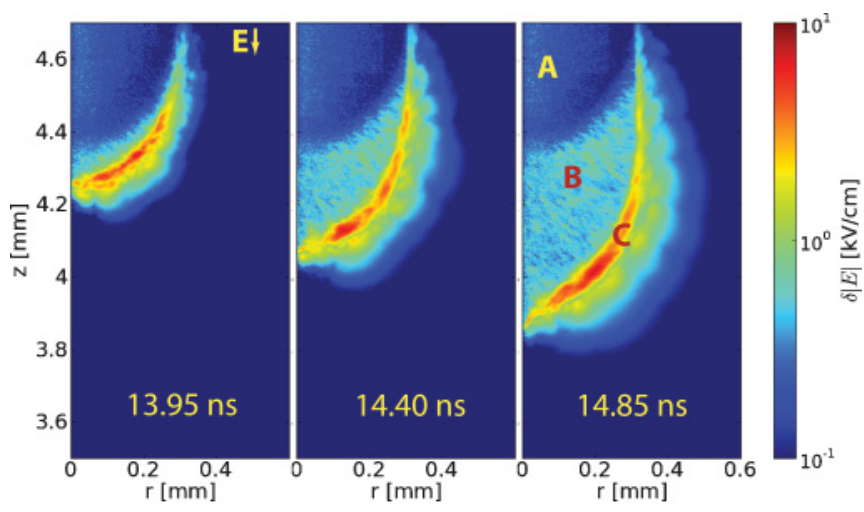

FIG. 3. (Color online) Highest deviation of the absolute value of the electric field from its azimuthal average at three snapshots. The volume labeled A corresponds to the evolution of the streamer up to $t=13.5 \mathrm{~ns}$, with noise switched off. When we switch on noise, the streamer body (B) develops low-amplitude, small-scale fluctuations. The streamer surface close to the tip (C) has fluctuations with a higher amplitude and an autocorrelation length of about one tenth of a millimeter. The amplitude grows, but in this simulation it remains small compared with the field values of some hundreds of kilovolts per centimeter in that area.

The deviation from symmetry can then be defined as

$$
\delta u=\max _{\theta}\left(u-\langle u\rangle_{\theta}\right) .
$$

Figure 3 shows $\delta|E|$ at three instants of time after noise is introduced into the simulation.

This simulation is highly demanding: the short streamer evolution represented in Fig. 3 took about five weeks using two dual-core 3-GHz AMD Opteron processors. We could not run the simulation long enough to observe actual branching; nevertheless, we can use the present result for a first quantitative estimation of the time needed to branch.

Let us look at the Fourier transform of the electron density $\sigma$ along the azimuthal coordinate $\theta$ :

$$
\tilde{\sigma}(r, z, k)=\frac{1}{2 \pi} \int_{0}^{2 \pi} d \theta n_{e}(r, z, \theta) e^{-i k \theta} .
$$

We can define the "total spectral content" of mode $k$ of the electron density as

$$
W_{k}=2 \pi \int_{-\infty}^{+\infty} d z \int_{0}^{\infty} d r r \tilde{\sigma}(r, z, k) .
$$

If the streamer is close to cylindrical symmetry (i.e., it is far from a branching state), $\left|W_{0}\right| \gg\left|W_{k}\right|$ for all $k>0$. Hence if we define $A=\sum_{k>0}\left|W_{k}\right|^{2} /\left|W_{0}\right|^{2}$ we can postulate that the condition for branching is $A \sim 1$.

\section{Extrapolating to streamer branching}

In Fig. 4 the value of $A$ during the simulated time frame is plotted as a black line. During the full evolution we find $A \ll 1$; i.e., the streamer was at all times far from branching. However we can use $A(t)$ to obtain a first estimation of the branching time. After a transient, $A$ growths exponentially; extrapolating this growth to $A \sim 1$ we can roughly estimate the branching time as $t_{\text {branch }} \sim 21 \mathrm{~ns}$. The streamer velocity is approximately 


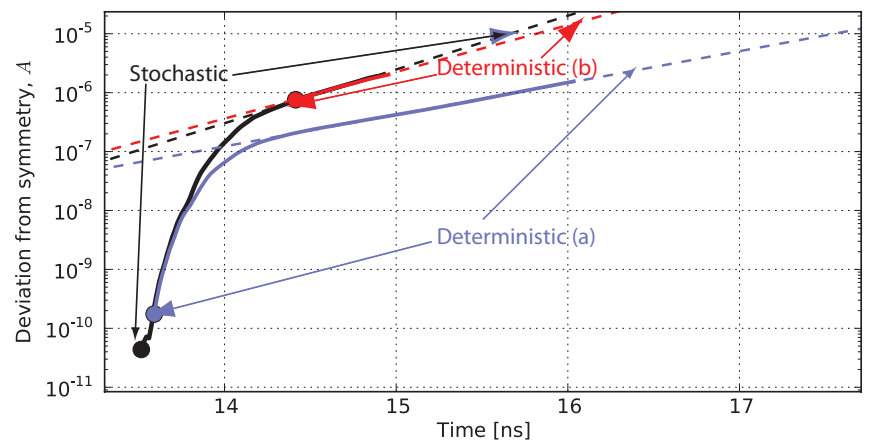

FIG. 4. (Color online) Evolution of $A$ as defined in the text. We show the evolution of three runs: in all runs the system was deterministic and cylindrically symmetrical up to a time of $13.5 \mathrm{~ns}$. After this point we add stochasticity and allow deviations from cylindrical symmetry. In the curves marked as deterministic, we remove noise again at the time marked with the dots while keeping the asymmetric perturbations that have evolved up to that time. Within this limited time frame, $A$ seems go through a transient, fast growth phase until it settles into an exponential growth. The dashed line fits this second phase. For the completely stochastic run the best fit is $A=a e^{t / \tau}$ with $a=5 \cdot 10^{-20}$ and $\tau=0.48 \mathrm{~ns}$. Extrapolating, this predicts branching at $t \sim-\tau \log a \approx 21 \mathrm{~ns}$.

$v=0.32 \mathrm{~mm} / \mathrm{ns}$ and hence after the introduction of noise the streamer would run for about $2.4 \mathrm{~mm}$ before branching.

\section{Comparison with experiment}

In Refs. [9,10], the streamer length between branching events is measured for positive streamers of varying diameters in air. The ratio of branching length over streamer diameter is always found to be about 12 to 15 . The streamer diameter is here taken from optical measurements; i.e., it is the radiative diameter that is estimated to be about half of the electrodynamic diameter [59]. With that estimation, our ratio of branching length over streamer diameter is about 8. One must also take into account that (a) short branching distances are harder to measure and therefore the average in Refs. $[9,10]$ may be slightly overestimated and (b) both in our model and in observations branching distance is random: we are comparing the result of only one simulation with an average over many measurements so a certain discrepancy seems natural.

\section{E. Testing the relevance of fluctuations}

Our estimation of the branching length is close to the measured value. But were the electron-density fluctuations relevant for this result? To test the relevance of a persisting noise for the growth of cylindrically perturbed modes, we performed two simulations in which noise was removed after some time. These are shown in the two curves of Fig. 4 labeled as deterministic (a) and (b). We see that noise always increases the growth rate of the deviations but also that even a relatively small amount of noise during a short time is enough to trigger an instability that would eventually lead to streamer branching.

\section{F. Discharges at lower air density like sprites}

Sprite discharges are streamerlike but develop in lower air densities at high altitudes in the atmosphere. They also branch frequently, as characterized, e.g., in Ref. [8]. At lower pressure, relatively more electrons are involved in the formation of physically similar streamers [6]; therefore electron-density fluctuations are weaker $\left(g^{-1 / 2} \approx 10^{-4}\right)$. This could simply result in a somewhat larger ratio between branching length and streamer diameter, but this quantity has not yet been measured in sprites.

If sprite branching statistics due to the natural intrinsic fluctuations is predicted as well as measured, and if actual sprite branching actually happens more frequently than predicted, then one has to search for additional external sources of noise like cosmic rays or atmospheric inhomogeneities [33]. Otherwise the intrinsic mechanisms and fluctuations fully explain branching.

\section{SUMMARY}

We have argued that streamer branching has to be triggered by a finite perturbation. In the quoted laboratory experiments in very clean artificial air (which is a pure nitrogen-oxygen mixture), these perturbations can only be inherited from the initial state, or they can be triggered by intrinsic electrondensity fluctuations. We have introduced such electron-density fluctuations into the common density model for streamer discharges in air, we have simulated the developing branching instability of positive streamers in three dimensions, and we have extrapolated the branching time and length from these simulations. Although longer simulations must be performed to check that the evolution suggested in Fig. 4 continues until the streamer branches, our extrapolation agrees quite well with the observations in artificial air. But streamer branching in ambient room air is quite similar to that in artificial air; therefore we predict the branching of streamers in ambient air as well. We find that the electron-density fluctuations in the leading edge of the streamer ionization front do contribute to triggering the instability. We predict that, due to the lower electrondensity fluctuations at lower air density, sprite discharges should branch somewhat less than streamers in air at ground level. If larger branching ratios are found, one needs to look for external sources of noise that could trigger this behavior.

\section{ACKNOWLEDGMENTS}

This work was supported by the Spanish Ministry of Science and Innovation, MICINN, under Project No. AYA200914027-C05-02, and by the Junta de Andalucia, Proyecto de Excelencia No. FQM-5965.
[1] H. Raether, Z. Phys. 112, 464 (1939).

[2] Y. P. Raizer, Gas Discharge Physics (Springer-Verlag, Berlin, 1991).
[3] R. C. Franz, R. J. Nemzek, and J. R. Winckler, Science 249, 48 (1990).

[4] V. P. Pasko, Nature (London) 423, 927 (2003). 
[5] V. P. Pasko, U. S. Inan, and T. F. Bell, Geophys. Res. Lett. 25 2123 (1998).

[6] U. Ebert, S. Nijdam, C. Li, A. Luque, T. Briels, and E. van Veldhuizen, J. Geophys. Res. 115, A00E43 (2010).

[7] G. J. J. Winands, Z. Liu, A. J. M. Pemen, E. J. M. van Heesch, K. Yan, and E. M. van Veldhuizen, J. Phys. D 39, 3010 (2006).

[8] M. G. McHarg, H. C. Stenbaek-Nielsen, T. Kanmae, and R. K. Haaland, J. Geophys. Res. 115, A00E53 (2010).

[9] T. M. P. Briels, E. M. van Veldhuizen, and U. Ebert, J. Phys. D 41, 234008 (2008).

[10] S. Nijdam, J. S. Moerman, T. M. P. Briels, E. M. van Veldhuizen, and U. Ebert, Appl. Phys. Lett. 92, 101502 (2008).

[11] S. Nijdam, F. M. J. H. van de Wetering, R. Blanc, E. M. van Veldhuizen, and U. Ebert, J. Phys. D 43, 145204 (2010).

[12] G. Wormeester, S. Pancheshnyi, A. Luque, S. Nijdam, and U. Ebert, J. Phys. D 43, 505201 (2010).

[13] G. Wormeester, S. Nijdam, and U. Ebert, Jpn. J. Appl. Phys., 08JA01 (2011).

[14] L. Niemeyer, L. Pietronero, and H. J. Wiesmann, Phys. Rev. Lett. 52, 1033 (1984).

[15] I. Gallimberti, J. Phys. D 5, 2179 (1972).

[16] I. Gallimberti, G. Bacchiega, A. Bondiou-Clergerie, and P. Lalande, C. R. Phys. 3, 1335 (2002).

[17] M. Akyuz, A. Larsson, V. Cooray, and G. Strandberg, J. Electrost. 59, 115 (2003).

[18] L. Loeb and J. Meek, The Mechanism of the Electric Spark (Stanford University Press, Palo Alto, 1941).

[19] M. Arrayás, U. Ebert, and W. Hundsdorfer, Phys. Rev. Lett. 88, 174502 (2002).

[20] C. Montijn, U. Ebert, and W. Hundsdorfer, Phys. Rev. E 73, 065401 (2006).

[21] N. Liu and V. P. Pasko, J. Phys. D 39, 327 (2006).

[22] E. D. Lozansky and O. B. Firsov, J. Phys. D 6, 976 (1973).

[23] B. Meulenbroek, A. Rocco, and U. Ebert, Phys. Rev. E 69, 067402 (2004).

[24] S. Tanveer, L. Schäfer, F. Brau, and U. Ebert, Physica D Nonlinear Phenomena 238, 888 (2009).

[25] C. Kao, F. Brau, U. Ebert, L. Schäfer, and S. Tanveer, Physica D Nonlinear Phenomena 239, 1542 (2010).

[26] U. Ebert et al., Nonlinearity 24, C1 (2011).

[27] P. G. Saffman and G. Taylor, Proc. R. Soc. London A 245, 312 (1958).

[28] A. Luque, F. Brau, and U. Ebert, Phys. Rev. E 78, 016206 (2008).

[29] M. Arrayás and U. Ebert, Phys. Rev. E 69, 036214 (2004).

[30] G. Derks, U. Ebert, and B. Meulenbroek, J. Nonlinear Sci. 18, 551 (2008).

[31] A. A. Kulikovsky, Phys. Rev. Lett. 89, 229401 (2002).

[32] S. V. Pancheshnyi and A. Y. Starikovskii, J. Phys. D 34, 248 (2001).

[33] A. Luque and F. J. Gordillo-Vázquez, Geophys. Res. Lett. 38, L04808 (2011).
[34] S. Nijdam, K. Miermans, E. M. van Veldhuizen, and U. Ebert, IEEE Trans. Plasma Sci. (2011) (published online, doi: 10.1109/TPS.2011.2158661).

[35] A. Luque, U. Ebert, and W. Hundsdorfer, Phys. Rev. Lett. 101, 075005 (2008).

[36] L. Papageorgiou, A. C. Metaxas, and G. E. Georghiou, J. Phys. D 44, 045203 (2011).

[37] N. Y. Babaeva and M. J. Kushner, IEEE Trans. Plasma Sci. 36, 892 (2008).

[38] N. Y. Babaeva and M. J. Kushner, J. Phys. D 42, 132003 (2009).

[39] S. Nijdam, G. Wormeester, E. M. van Veldhuizen, and U. Ebert, e-print arXiv:1108.1032.

[40] T. M. P. Briels, J. Kos, G. J. J. Winands, E. M. van Veldhuizen, and U. Ebert, J. Phys. D 41, 234004 (2008).

[41] S. Nijdam, C. G. C. Geurts, E. M. van Veldhuizen, and U. Ebert, J. Phys. D 42, 045201 (2009).

[42] C. Li, U. Ebert, and W. Hundsdorfer, J. Comput. Phys. (2011) (published online, doi: 10.1016/j.jcp.2011.07.023).

[43] G. D. Moss, V. P. Pasko, N. Liu, and G. Veronis, J. Geophys. Res. 111, A02307 (2006).

[44] O. Chanrion and T. Neubert, J. Comput. Phys. 227, 7222 (2008).

[45] O. Chanrion and T. Neubert, J. Geophys. Res. 115, A00E32 (2010).

[46] C. Li, U. Ebert, and W. Hundsdorfer, J. Phys. D 42, 202003 (2009).

[47] C. Li, W. Hundsdorfer, and U. Ebert, e-print arXiv:1101.1189.

[48] C. Li, U. Ebert, and W. Hundsdorfer, J. Comput. Phys. 229, 200 (2010).

[49] M. Arrayás, J. P. Baltanás, and J. L. Trueba, J. Phys. D 41, 105204 (2008).

[50] U. Ebert, W. van Saarloos, and C. Caroli, Phys. Rev. E 55, 1530 (1997).

[51] U. Ebert and W. van Saarloos, Physica D Nonlinear Phenomena 146, 1 (2000).

[52] C. Gardiner, Handbook of Stochastic Methods: For Physics, Chemistry and the Natural Sciences (Springer-Verlag, Berlin, 2004).

[53] U. Ebert, C. Montijn, T. M. P. Briels, W. Hundsdorfer, B. Meulenbroek, A. Rocco, and E. M. van Veldhuizen, Plasma Sources Sci. Technol. 15, 118 (2006).

[54] A. Luque, U. Ebert, C. Montijn, and W. Hundsdorfer, Appl. Phys. Lett. 90, 081501 (2007).

[55] C. Montijn, W. Hundsdorfer, and U. Ebert, J. Comput. Phys. 219, 801 (2006).

[56] See Supplemental Material at http://link.aps.org/supplemental/ 10.1103/PhysRevE.84.046411 for a complete movie of this simulation.

[57] N. Liu and V. P. Pasko, J. Geophys. Res. 109, A04301 (2004).

[58] A. Luque, V. Ratushnaya, and U. Ebert, J. Phys. D 41, 234005 (2008).

[59] S. Pancheshnyi, M. Nudnova, and A. Starikovskii, Phys. Rev. E 71, 016407 (2005). 